\title{
Navigation of a Rolling Microrobot in Cluttered Environments for Automated Crystal Harvesting
}

\section{Conference Paper}

Author(s):

Charreyron, Samuel (D); Pieters, Roel S.; Tung, Hsi-Wen; Gonzenbach, Maurice; Nelson, Bradley J.

Publication date:

2015

Permanent link:

https://doi.org/10.3929/ethz-b-000104569

Rights / license:

In Copyright - Non-Commercial Use Permitted

Originally published in:

https://doi.org/10.1109/IROS.2015.7353371 


\title{
Navigation of a Rolling Microrobot in Cluttered Environments For Automated Crystal Harvesting
}

\author{
Samuel L. Charreyron, Roel S. Pieters, Hsi-Wen Tung, Maurice Gonzenbach, and Bradley J. Nelson
}

\begin{abstract}
We present a holistic system for automating the motion of a rolling microrobot for protein crystal harvesting. The RodBot, which was introduced in previous work, is able to perform noncontact manipulation of microscopic objects such as fragile crystals by trapping them in an induced vortex fluid flow. Here, we are concerned with navigating the RodBot autonomously in a liquid environment containing obstacles such as crystals. A literature review shows existing approaches to untethered microrobot control are limited and cluttered environments are often not considered. We demonstrate real-time tracking of the RodBot and surrounding obstacles, kinematic obstacle-free path planning, and nonholonomic path following. The system was evaluated in qualitative and quantitative experiments, shows satisfactory performance, and presents itself as a first step towards fully automated crystal harvesting.
\end{abstract}

\section{INTRODUCTION}

Microrobotics has seen significant strides in device characterization, fabrication, and novel actuation methods such as optical [1], chemical [2], or biological [3]. Such advances allow for a better understanding of microrobots can function efficiently at small-scales, given the different balances of forces they encounter. Simultaneously, new fabrication techniques allow for increased device sophistication and capability.

A promising application of micorobots is the manipulation of objects that may be too small or too delicate for a human to handle manually. Micromanipulation has been proposed for such applications as micro-machining [4], cell manipulation [1], and MEMS microassembly [5]. In [6], [7], a rolling microrobot design called the RodBot was introduced for use in $\mathrm{X}$-ray crystallography X-ray crystallography concerns the study of properties of biological molecules and proteins, which are crystallized and analyzed by small-angle x-ray scattering, and is used extensively in the pharmaceutical industry [8].

Traditional methods for retrieving crystals from their growth sites require skilled operators to manually extract crystals with a precise tool. Protein crystals, which often have dimensions of the order of $100 \mu \mathrm{m}$, are difficult to manipulate manually, given that average human tremor for experienced surgeons is of the order of $100 \mu \mathrm{m}$ [9]. The RodBot enables non-contact retrieval of the fragile crystals thus allowing for easy and precise transportation, while limiting the potential for damage during the extraction process.

An additionally attractive prospect is that of fully automated crystal harvesting. This would allow for considerable

S. Charreyron (samuelch@ethz.ch), R. Pieters (rpieters@ethz.ch), B. J. Nelson (bnelson@ethz.ch) are with the Multi-Scale Robotics Lab, ETH Zurich, Tannenstrasse 3, Zurich, 8092, Switzerland. time and cost savings by enabling less-skilled operators to oversee large-scale harvesting operations. Developing algorithms for microrobot automation in general is still an open and under-explored topic of research [10]. Many fundamental automation topics to be addressed include but are not limited to robust tracking of the microrobotic tool, perception of the microrobot's environment, automation of the microrobot's locomotion, automation of manipulation tasks, high-level coordination and scheduling of manipulation tasks.

In this paper, we address the first three tasks outlined above. We describe a complete vision-based motion control system for the RodBot during automated crystal harvesting. We show that its locomotion can be automated, even in cluttered environments containing obstacles such as air bubbles or crystals. We show a strategy and functional methods for detecting and mapping such obstacles in the environment, planning of obstacle-free motion, and execution of motion plans.

\section{A Brief ReView of Existing Approaches to Unthethered Microrobot Motion Automation}

\section{A. Motion Planning}

Planning strategies including heuristic-search [11] and sampling-based methods [12] can be found in microassembly and cell-manipulation literature. For a review of planning and control approaches to micromanipulation, refer to [13]. In our review of the literature, we only found two attempts at advanced kinematic path planning of untethered microrobots other than simple waypoint selection. The first is in [14], where an optimal path was generated to limit the control effort required to move a microrobot through pulsing blood flow. In [15], the fast marching method (FMM) was used to solve the path planning problem between a start and end position in a blood vessel.

\section{B. Motion Control}

Simpler point-to-point control strategies can be found in [16], [17]. These require the microrobot to move to the vicinity of a desired position and do not take into account the path that the robot must take to reach the goal. Pointto-point strategies may not be applicable to nonholonomic systems such as Artificial Bacterial Flagella (ABF) [18], rolling microrobots, or microjets, since there may not be a feasible path between two adjacent waypoints.

Robust Control can be used with multiple-input multipleoutput (MIMO) systems to ensure guarantees on a plant's performance, given a bounded disturbance model and 
bounded uncertainties in the plant model. In [19], the authors used $H_{\infty}$ control to achieve 3D motion control for a magnetic body actuated with magnetic field gradients. This approach assumes linear drag forces in low Reynolds number fluid regimes and a fully-actuated system and is therefore not applicable to nonholonomic microrobots.

It can be difficult to develop accurate kinematic models for a microrobot due to the uncertainty in model parameters. Adaptive schemes can be used to simultaneously estimate the parameter values online, and guarantee convergence to an equilibrium or trajectory. In [20] time delay estimation (TDE) was used to account for uncertainty in parameters associated with nonlinear dynamics in the system model. In [14], nonlinear adaptive control was considered for a magnetically-pulled microrobot navigating through endovascular blood vessels. Backstepping was used to generate a control law that is Lyapunov-stable about a trajectory given linear uncertain parameters in the dynamics. Convergence was demonstrated in simulations, but not in practical experiments. Additionally, not all parameters such as non-linear drag force can be captured by such an approach.

In [15], model predictive control (MPC) was proposed to add robustness to microrobot motion control. In this work, the microrobot consisted of a magnetically-pulled magnetic bead moving through the endovascular system and was both actuated and tracked by magnetic resonance imaging (MRI). MPC functions by predicting system outputs based on a model of the dynamics and solving an optimization problem over a finite time window projected into the future. It can therefore be beneficial in anticipating sharp changes in the control input such as those required to navigate through a blood vessel bifurcation [21]. Nevertheless, the computational requirements associated with solving the optimization problem at every time step may be prohibitively expensive for fast control.

\section{Automation System Design}

\section{A. Physical System}

The physical system consists of 1) a torque driven sub$\mathrm{mm}$ rolling microrobot 2) a magnetic field generator (MFG) and control box which generate the fields required to drive the microrobot and comprises the actuation system 3) a camera-mounted microscope providing visual feedback 4) a computer platform which controls the MFG based on visual feedback.

1) The RodBot: The RodBot is a $300 \times 60 \times 50 \mu \mathrm{m}$ rectangularly shaped microrobot consisting of a biocompatible polymer SU-8 body and a number of transversally magnetized CoNi posts evenly spaced along its body. When a magnetic field is applied to the workspace, the magnetized posts align with the applied field and rotate the robot's body. A rotating magnetic field causes the RodBot to rotate about its long axis, allowing it to roll along a substrate surface similar to a rolling-pin. The RodBot operates in low Reynolds number liquid environments and its forward velocity depends on the viscosity of the surrounding fluid. By setting the field direction and rotation frequency, the orientation and speed of

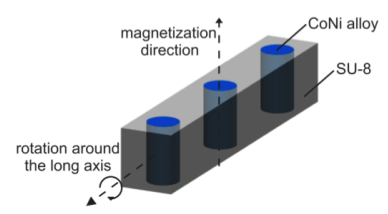

(a)

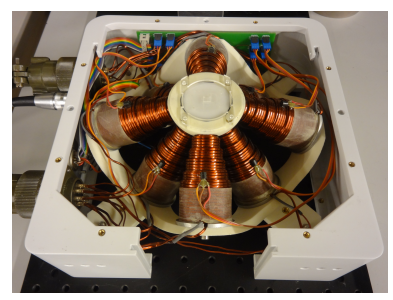

(b)
Fig. 1. (a) The RodBot, a rectangular rolling microrobot design (b) The MFG, an 8-coil magnetic field generator designed for the actuation of sub$\mathrm{mm}$ and sub- $\mu \mathrm{m}$ devices.

the RodBot can be controlled respectively. Thus the RodBot's rolling kinematics can be represented with a nonholonomic unicycle-like model. The RodBot is depicted in Fig. 1a.

2) The MFG: The RodBot is actuated by a multi-coil magnetic field generator suited for sub-mm and sub- $\mu \mathrm{m}$ devices. The latter was engineered so that it is easily integrated with regular and inverted optical microscopes. The MFG can generate fields in the $\mathrm{mT}$ range over a spherical working area with a radius of $10 \mathrm{~mm}$ and dynamic fields up to $2 \mathrm{kHz}$ [22]. The MFG is shown in Fig. $1 \mathrm{~b}$.

3) The Automation Loop: The final portion of the physical system is a desktop computer which runs the visual servo automation loop. A Basler scA-780fm camera (Basler AG) is mounted to the microscope and connected to the computer via a Firewire interface. The automation loop is split into three functional modules: a) computer vision for tracking the microrobot and detecting obstacles in its environment b) a motion planner to generate optimal obstacle-free path between a start and goal pose c) a motion controller performs visual servoing to drive the RodBot along the generated path.

\section{B. Magnetic Actuation}

Magnetic forces and torques can be applied independently to magnetically functionalized microrobots by the controlling the direction of magnetic fields and their spatial gradient [23] using magnetic manipulation systems such as the MFG. When immersed in a magnetic field $\mathbf{B}$, a magnetic body experiences a torque $\mathbf{T}_{m}$ which tends to align the material magnetization with the applied field direction in (1).

$$
\mathbf{T}_{m}=\mathbf{M} \times \mathbf{B}
$$

In a multi-coil setup, assuming ideal soft-magnetic coils operating in their linear regions and negligible hysteresis, the magnetic field flux density at position $P: B(P)$ can be decomposed into linear terms $B_{1}(P) \cdots B_{n}(P)$ corresponding to the magnetic field generation effect of each individual coil at a unit current. Thus a $3 \times n$ field contribution matrix $B(P)$ exists for every multi-coil setup and relates the currents running through each coil and the induced magnetic field.

$$
B(P)=\left[\begin{array}{lll}
B_{1}(P) & \cdots & B_{n}(P)
\end{array}\right]\left[\begin{array}{c}
i_{1} \\
\vdots \\
i_{n}
\end{array}\right]=\mathcal{B}(P) I
$$


One can induce dynamic magnetic fields such as stepping and rotating fields over the workspace by varying the electromagnet coil currents over time [24].

\section{Computer Vision}

1) Tracking the RodBot: The RodBot is tracked in realtime by detection of its dark magnetic posts. The input image is first pre-processed by performing adaptive thresholding to remove sensitivity to varying illumination over the image, and morphological closing is used to split the posts into disjoint blobs. Simple blob detection is used for feature extraction, and blobs are filtered by size to reduce the number of extracted features. Because the feature extraction step generally produces a large number of features, heuristicbased filtering is used for selecting relevant features. In the case of the RodBot, two visual heuristics are used in the feature selection step. The first is the number of magnetic posts which is known a priori. The second is that the posts should appear along a straight line and should be evenly spaced, as shown in Fig. 2.
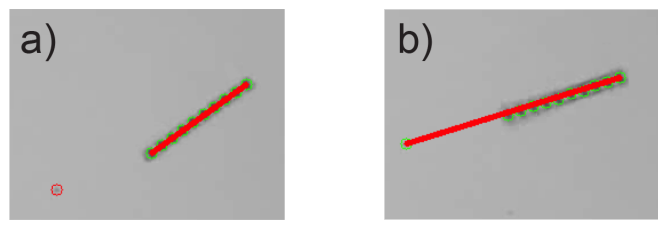

Fig. 2. Feature selection heuristics based on the RodBot appearance. Features are rejected if they are not (a) colinear with other features or (b) not equally spaced.

First a calibration step determines the spacing between magnetic posts. Thereafter, features $p_{k}$ are given a score penalizing orthogonal distance to the colinear line $d_{\perp}\left(p_{k}\right)$ and transversal distance to the supposed location $d_{\|}\left(p_{k}\right)$. Since only one feature can be kept per post, the feature with a maximum score is kept in the consensus set $\chi_{i}$. The total score of the consensus set is shown in (3). In this equation $d x$ refers to the true spacing between magnetic posts, while $\left|\chi_{i}\right|$ represents the cardinality of the consensus set.

$$
C\left(\chi_{i}\right)=\left|\chi_{i}\right|-\sum_{p_{k} \in \chi_{i}} \frac{d_{\perp}\left(p_{k}\right)}{d x^{2}}+2 \frac{d_{\|}\left(p_{k}\right)}{d x}
$$

The previous also provides a confidence measure as to the accuracy of tracking. If the confidence exceeds a certain threshold, the RodBot is assumed to be tracked accurately.

2) Tracking Obstacles: It is considerably difficult to visually define what constitutes an obstacle, given they can take on almost infinite appearances. Nevertheless, a general approach was considered to flexibly detect physical obstacles. The detection scheme is implemented, as a proof of concept, for two types of obstacles: air bubbles and protein crystals.

At microscopic scales, capillary forces between liquidair interfaces become much more dominant than at larger scales. Due to such strong adhesive forces, microrobots may not have enough propulsive force to detach themselves if they happen to come into contact with bubbles. In Fig. 3a, we show a group of air bubbles in the range of several hundred $\mu \mathrm{m}$ in diameter formed in a solution of isopropyl alcohol. They are easily detected by performing the following sequence of image processing steps. Noise is reduced by performing heavy blurring with a 5 pixel box filter. The box filter has the added feature of being conservative since it expands the obstacle-regions such that they cover slightly more area than the bubbles do in reality. The image is thresholded and the edges of the bubbles are detected with a Canny filter [25].

In automated crystal harvesting, the RodBot would need to sequentially retrieve crystals from a dish containing a potentially large number of crystals and deposit them in a retrieval site. Therefore accurate detection of the crystals would be required, such that a path to the next crystal to be retrieved could be planned, while avoiding the other crystals in the dish. A similar procedure is used to detect the crystals shown in Fig. 3d. Blurring is performed, followed by adaptive thresholding to account for the varying intensity of the crystal's edges. The edges are grown using a morphological closing operation to ensure that they form closed contours. Finally, contours are extracted using OpenCV's contour detection algorithm [26] and are filtered by area so that they are large enough to represent protein crystals (minimum $0.05 \mu \mathrm{m}^{2}$ ).

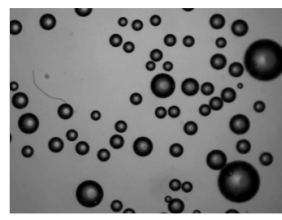

(a)

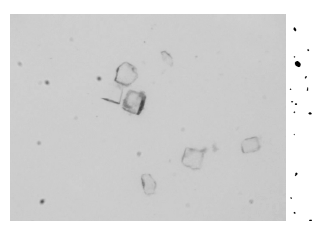

(d)

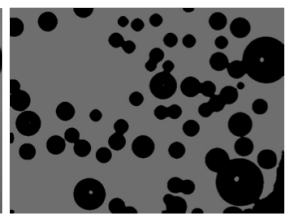

(b)

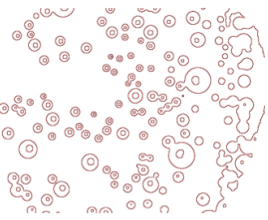

(c)

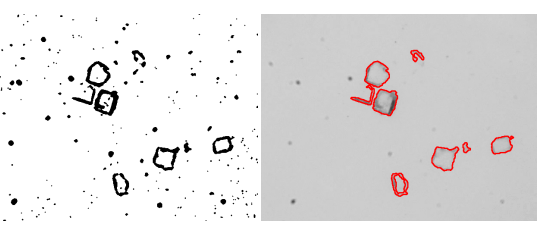

(e)

Fig. 3. Top: image processing steps performed in detecting sub-millimeter diameter air bubbles. (a) original image (b) binary thresholding (c) canny edge detection. Bottom: image processing steps for detecting protein crystals in a dish of buffer solution. (d) original image (e) adaptive thresholding and morphological closing (f) contour detection and filtering.

\section{Motion Planning}

Rather than use a sampling-based method such as probabilistic roadmaps or rapidly exploring random trees - randomized methods which are often resorted to in higher dimensional problems [27] - a deterministic approach was used. The planning strategy used was search or lattice-based planning which is implemented in the search-based planning library (SBPL) [28]. While the memory requirement of lattice-based planning scales linearly with the discretization size, the state-space dimensionality, and the size of the environment, it can yield optimal, complete, and kinematically feasible solutions given an adequate discretization. 
In lattice-based planning [28], the robot's environment is discretized and represented in a graph structure $G(V, E)$ where the vertices $V$ represent discrete states in the configuration or $\mathcal{C}$-space and the edges $E$ represent small kinematic transitions between such states, also known as motion primitives. Traditional graph-search algorithms such as Dijkstra's algorithm or $\mathrm{A}^{\star}$ can then be used to find a path between two configurations. As with roadmap-based methods, there is an inherent decoupling between the environment representation and path planning queries. Thus, general search algorithms at the query stage can be used in a vast variety of problems.

1) Defining the Environment: We introduce the following discretization for the $\mathcal{C}$-space. The portion of the workspace that can be seen in the camera view is divided into square cells of side length $25 \mu \mathrm{m}$. The upper-bound on the cell size is that it must be fine enough to capture the geometry of the obstacle regions. The orientation is discretized into sixteen possible angles. The RodBot is given a rectangular geometrical footprint of $300 \mu \mathrm{m}$ x $50 \mu \mathrm{m}$ in correspondence with its real dimensions. The geometrical environment is loaded from a binary image obtained by the obstacle tracker unit. Configurations in which the the RodBot collides with the obstacle region - where at least one of the footprint's points lay within the obstacle region - are marked as invalid states.

2) Motion Primitives: Five possible forward motion primitives were defined for the RodBot namely straight motion, two large turning arcs, and two smaller turning arcs. Other primitives such as backwards motion are possible but were not investigated in this work.

3) Solving the Planning Problem: Performing heuristic search on graphs containing millions of nodes can be a computationally expensive and slow process. The weighted$\mathrm{A}^{*}$ variation sacrifices optimality for faster computation by inflating the heuristic lower bound by $\epsilon$, a positive factor: $f(n)=g(n)+(1+\epsilon) h(n)$. Here $f(n)$ is the heuristic for node $n, g(n)$ represents the accumulated cost of traveling from the start node to node $n$, and $h(n)$ a guaranteed lower bound on the cost from $n$ to the goal node.

We used the anytime dynamic variation of $\mathrm{A}^{*}$ from [28], performing a sequence of weighted- $A^{*}$ searches with an $\epsilon$ decreasing towards 1 . Thus, provided a small initial time has elapsed, a solution can always be returned at the algorithm's termination.

\section{E. Path-following Control}

Path-following is done in closed-loop by visually tracking the RodBot's position and acting on the two available control inputs, orientation and forward velocity.

1) RodBot Kinematics: The RodBot's kinematics can be approximated with the standard unicycle-model. The RodBot has two controllable inputs which are its forward velocity $v$ and orientation $\theta_{c}$, while it operates in the $\operatorname{SE}(2)$ space of dimension 3 , thus showing the nonholonomic nature of its kinematics.

2) Virtual Target Based Path Follower: In our pathfollowing control strategy, nonholonomic control is ensured by driving to a point $\mathbf{P}(s)$ along the kinematically feasible preplanned path. Thus the RodBot is set to point towards the target point by adjusting the orientation of the magnetic field. The velocity can be adjusted proportionally to the goal distance, such that the robot slows down near the goal. This is shown in (4), where e represents a vector between the current position $\mathbf{R}$ and target position $\mathbf{P}(s)$ along the parametrized desired path $\tau(s), s \in[0,1]$. The forward velocity $v$ is proportional to the target distance by a tuned parameter $k_{v}$ and set by the field rotation frequency $\omega_{m}$. Note that to prevent input saturation, it is scaled by a arctan sigmoid function such that it never exceeds $v_{\max }$.

$$
\left\{\begin{array}{l}
v \\
\theta_{c}=L \mathbf{e} \\
\ddot{s} \quad k_{d}(\|\mathbf{e}\|-d)
\end{array}\right.
$$

To overcome the problems associated with projecting the RodBot's position on the desired path, a moving virtual target is used, as introduced in [29]. By driving to a point forward along the path, one avoids the scenario shown in Fig. 4a, where sideways motion is required. In this scheme, we define the target to be a point or cart moving along the desired path. The moving virtual target can be seen in Fig. 4b. An artificial third input is introduced, the cart's acceleration $\ddot{s}$, which is controlled independently and is proportional to the difference between $d$, a desired distance between itself and the robot, and the actual distance as measured by the tracker.

Thus the cart attempts to maintain a constant separation between itself and the RodBot while the RodBot tries to move towards the target. An intuitive way to visualize this is by imagining a cart that is connected to the RodBot via an elastic rope and that drags it along the desired path.

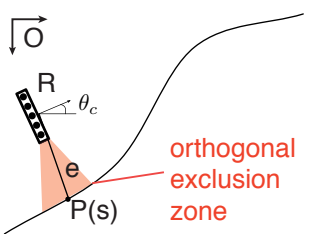

(a)

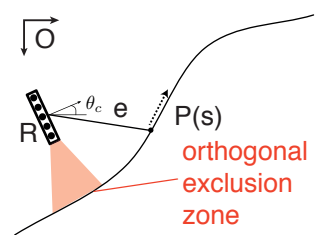

(b)
Fig. 4. In (a) the problem with driving to the nearest point on the path, which may be physically unrealizable due to stiction. In (b) this is resolved by instead driving to a point $\mathbf{P}(s)$ which moves forward along the path.

\section{EXPERIMENTAL EVALUATION}

\section{A. Quantitative Evaluation of Obstacle-free Motion}

Because the presence of obstacles is decoupled from the path-following task, an obstacle-free characterization of the system performance was performed. In order to test the motion performance of the system for both straight-line path segments and tight turns, experiments were conducted at various magnetic field rotation frequencies $\omega_{m}$ along the figureeight paths of Fig. 5. Different virtual target separations $d$ were also tested to show their effect on motion at higher velocities. 


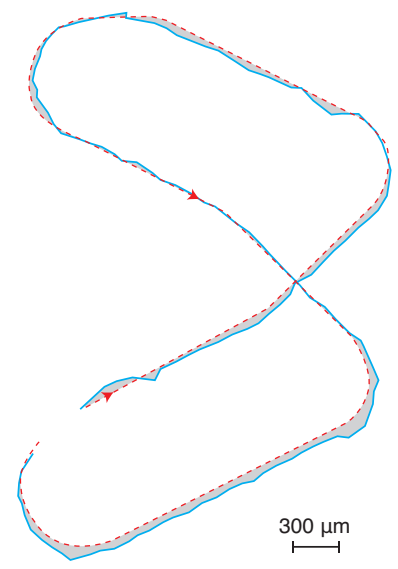

(a) $\omega_{m}=1 \mathrm{~Hz}, d=75 \mu \mathrm{m}$

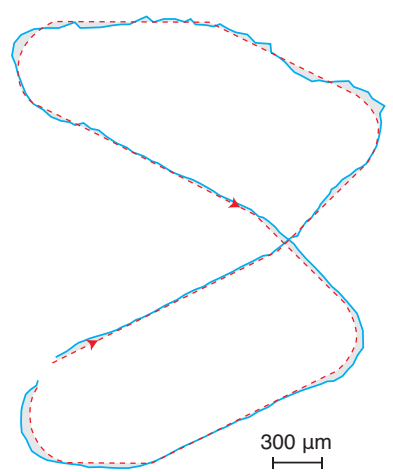

(b) $\omega_{m}=5 \mathrm{~Hz}, d=180 \mu \mathrm{m}$

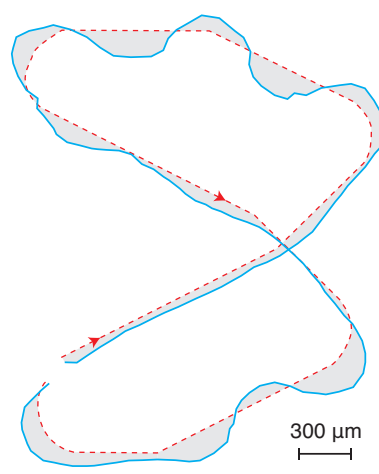

(c) $\omega_{m}=9 \mathrm{~Hz}, d=180 \mu \mathrm{m}$

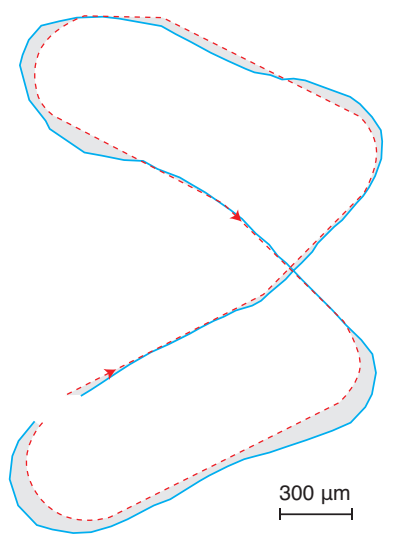

(d) $\omega_{m}=10 \mathrm{~Hz}, d=250 \mu \mathrm{m}$

Fig. 5. Motion experiments at varying velocities and virtual target distances on an obstacle-free figure 8 Path. The path was generated with four waypoints with angles at multiples at $\pi / 4$. The actual RodBot position was tracked manually frame-by-frame. The desired path is dotted and the traveled path is shown in solid. The area between the desired and traveled paths is filled in grey.

Validation of the RodBot's position was performed by manual frame-by-frame inspection of the captured videos. As a measure of performance, the area $A$ between the desired path $\rho$ the actual path $p$ traversed by the robot was calculated. This area was normalized to the desired path length $l(\rho)$ to form a scoring metric $S$ as shown in (5). The average velocity was also calculated by measuring the time taken to traverse the path $p$ in seconds. Note that the distances were calibrated by comparing pixel distances with the RodBot's long edge, assumed to be $300 \mu \mathrm{m}$ long. Numerical data from the experiments is listed in Table I.

$$
S=\frac{A(\rho, p)}{l(\rho)} \quad v_{a v g}=\frac{l(p)}{t}
$$

1) Results at Low Velocities: At low rotation frequencies and low velocities, namely below $\omega_{m}=5 \mathrm{~Hz}$, precise motion could be observed with relatively little deviation from the desired path as can be seen in Fig. 5a and Fig. 5b. The grey areas, showing the deviation between the desired and actual motion, are relatively small. One can see a small steady-state error in the straight-line segments and negligible oscillation. Both can be attributed to errors when tracking the RodBot's center point, as well as some jagged motion possibly due to irregularities in the manufacturing of the RodBot and in the bottom surface smoothness. Using the scoring function shown in (5), both experiments show very similar performance in motion control.

2) Results at High Velocities: Note that for higher velocities in the second trial, the distance of the virtual target was increased. This was done to increase robustness and ensure that the virtual target always stayed a safe distance ahead of the RodBot. If the RodBot came too close to or passed the virtual target, it was prone to undesirable behavior such as back and forth motion or rotation around the virtual target.

While the tracking and motion control of the RodBot was quite satisfactory at velocities less than $600 \mu \mathrm{m} / \mathrm{s}$, undesirable motion became quite noticeable at higher velocities greater than $900 \mu \mathrm{m} / \mathrm{s}$. In Fig. 5c, one can see considerable overshoot which induces oscillation following the sharp corner turns in the path. Increasing the separation distance $d$ between the RodBot and virtual target increased robustness when negotiating sharp corners. An explanation is that the increased distance allowed the RodBot to better anticipate the sharp turns. Because the tracker was further ahead, the RodBot started turns earlier and was able to follow the tight arcs. In the fourth experiment with an increased distance, the RodBot demonstrated much smoother motion as can be seen in Fig. 5d.

TABLE I

NUMERICAL RESULTS FROM THE MOTION EXPERIMENTS SHOWN IN FIG. 5.

\begin{tabular}{c|cccr}
\hline Trial & $\omega_{m}(\mathrm{~Hz})$ & $d(\mu \mathrm{m})$ & $v_{\text {avg }}(\mu \mathrm{m} / \mathrm{s})$ & $\mathrm{S}(\mu \mathrm{m})$ \\
\hline 1 & 1 & 75 & 187 & 19.6 \\
2 & 5 & 180 & 629 & 19.4 \\
3 & 9 & 180 & 943 & 65.6 \\
4 & 10 & 250 & 820 & 32.9 \\
\hline
\end{tabular}

\section{B. Motion in Cluttered Environments}

Experiments in cluttered environments containing crystals were performed, showing the system's ability to plan obstacle-free, kinematically-feasible paths between crystals. This would be required when sequentially retrieving crystals from their growing dish and depositing them in a area suitable for collection. One can see accurate detection of the crystals, as well as tracking of the RodBot as it travels along its desired path. Two representative examples are depicted in Fig. 6.

\section{CONCLUSION}

We presented a system for automating the navigation of a nonholonomic rolling microrobot for crystallographic applications. Kinematically feasible obstacle-free paths between crystals are planned autonomously, and are executed by performing visual closed-loop path following. A literature 

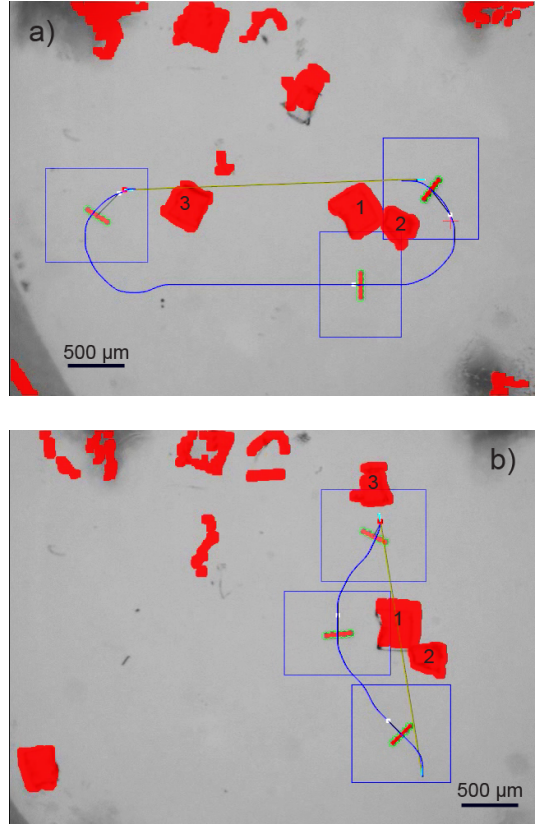

Fig. 6. Composite images representing the motion of a RodBot in environments containing crystals. The RodBot is marked with a blue rectangle and the detected crystals are shown in red. The automatically planned path is shown in blue. (a) Motion from the crystals 1,2 to crystal 3 (b) Motion to crystal 3 while avoiding crystals 1,2.

review showed limited approaches to motion planning and nonholonomic control of untethered microrobots. This is a first step towards full automation of crystal harvesting, solving one of the lower-level tasks of navigation in cluttered environments. Areas of future work include automating the retrieval and transport of crystals and coordination of such lower-level tasks.

\section{REFERENCES}

[1] P. Y. Chiou, A. T. Ohta, and M. C. Wu, "Massively parallel manipulation of single cells and microparticles using optical images," Nature, vol. 436, no. 7049, pp. 370-372, 072005.

[2] I. Khalil, V. Magdanz, S. Sanchez, O. Schmidt, and S. Misra, "The control of self-propelled microjets inside a microchannel with timevarying flow rates," IEEE Trans. Robot., vol. 30, no. 1, pp. 49-58, Feb. 2014.

[3] B. Behkam and M. Sitti, "Towards hybrid swimming microrobots: Bacteria assisted propulsion of polystyrene beads," in 28th Anпи. Int. Conf. of the IEEE EMBS, Aug 2006, pp. 2421-2424.

[4] R. Pelrine, A. Wong-foy, B. McCoy, D. Holeman, R. Mahoney, G. Myers, J. Herson, and T. Low, "Diamagnetically levitated robots: An approach to massively parallel robotic systems with unusual motion properties," in 2012 IEEE Int. Conf. Robot. Autom., May 2012, pp. 739-744.

[5] B. Tamadazte, E. Marchand, S. Dembélé, and N. Le Fort-Piat, "Cad model-based tracking and 3d visual-based control for mems microassembly," The International Journal of Robotics Research, vol. 29, no. 11, pp. 1416-1434, 2010.

[6] H. Tung, K. Peyer, D. Sargent, and B. Nelson, "Noncontact manipulation using a transversely magnetized rolling robot," Appl. Physics Lett., no. 103, Sep. 2013.

[7] R. Pieters, H. Tung, D. Sargent, and B. Nelson, "Non-contact manipulation for automated protein crystal harvesting using a rolling microrobot," in 19th IFAC World Congress, Cape Town, South Africa, 2014
[8] A. L. Carvalho, J. Trincão, and M. J. Romão, "X-ray crystallography in drug discovery," in Ligand-Macromolecular Interactions in Drug Discovery, ser. Methods in Molecular Biology, A. C. A. Roque, Ed. Humana Press, 2010, vol. 572, pp. 31-56.

[9] C. Riviere, R. Rader, and P. Khosla, "Characteristics of hand motion of eye surgeons," in Engineering in Medicine and Biology Society, 1997. Proceedings of the 19th Annual International Conference of the IEEE, vol. 4, Oct 1997, pp. 1690-1693 vol.4.

[10] E. Diller and M. Sitti, "Micro-scale mobile robotics," Foundations and Trends in Robotics, vol. 2, no. 3, pp. 143-259, 2013.

[11] D. Cappelleri, M. Fatovic, and U. Shah, "Caging micromanipulation for automated microassembly," in 2011 IEEE Int. Conf. Robot. Autom. (ICRA), May 2011, pp. 3145-3150.

[12] T. Ju, S. Liu, J. Yang, and D. Sun, "Apply rrt-based path planning to robotic manipulation of biological cells with optical tweezer," in 2011 Int. Conf. Mechatronics and Autom. (ICMA), Aug 2011, pp. 221-226.

[13] A. Banerjee and S. Gupta, "Research in automated planning and control for micromanipulation," IEEE Trans. Autom. Sci. Eng., vol. 10, no. 3, pp. 485-495, July 2013.

[14] L. Arcese, M. Fruchard, and A. Ferreira, "Adaptive controller and observer for a magnetic microrobot," IEEE Trans. Robot., vol. 29, no. 4, pp. 1060-1067, Aug 2013.

[15] K. Belharet, D. Folio, and A. Ferreira, "Endovascular navigation of a ferromagnetic microrobot using mri-based predictive control," in 2010 IEEE/RSJ Int. Conf. Intelligent Robots and Syst. (IROS), Oct. 2010, pp. 2804-2809.

[16] F. Temel, A. Bezer, and S. Yesilyurt, "Navigation of mini swimmers in channel networks with magnetic fields," in 2013 IEEE Intern. Conf. Robot. and Autom. (ICRA), May 2013, pp. 5335-5340.

[17] I. Khalil, M. P. Pichel, L. Abelmann, and S. Misra, "Closed-loop control of magnetotactic bacteria," Int. J. Robot. Res., vol. 32, no. 6 , pp. 637-649, 2013

[18] L. Zhang, J. J. Abbott, L. Dong, B. E. Kratochvil, D. Bell, an B. J. Nelson, "Artificial bacterial flagella: Fabrication and magnetic control," Applied Physics Letters, vol. 94, no. 6, p. 064107, January 2009. [Online]. Available: http://link.aip.org/link/?APL/94/064107/1

[19] H. Marino, C. Bergeles, and B. Nelson, "Robust electromagnetic control of microrobots under force and localization uncertainties," IEEE Trans. Autom. Sci. Eng., vol. 11, no. 1, pp. 310-316, Jan. 2014.

[20] A. Ghanbari, P. Chang, B. Nelson, and H. Choi, "Electromagnetic steering of a magnetic cylindrical microrobot using optical feedback closed-loop control," Intern. J. Optomechatronics, vol. 8, no. 2, pp. 129-145, 2014.

[21] K. Belharet, D. Folio, and A. Ferreira, "Untethered microrobot control in fluidic environment using magnetic gradients," in 2012 Int. Symp. Optomechatronic Tech., Oct 2012, pp. 1-5.

[22] B. Kratochvil, M. Kummer, S. Erni, R. Borer, D. Frutiger, S. Schürle, and B. Nelson, "Minimag: a hemispherical electromagnetic system for 5-dof wireless micromanipulation," in Experimental Robotics. Springer, 2014.

[23] J. Abbott, O. Ergeneman, M. Kummer, A. Hirt, and B. Nelson, "Modeling magnetic torque and force for controlled manipulation of soft-magnetic bodies," IEEE Trans. Robot., vol. 23, no. 6, pp. 1247 1252, Dec. 2007.

[24] K. E. Peyer, L. Zhang, and B. Nelson, "Bio-inspired magnetic swimming microrobots for biomedical applications," Nanoscale, vol. 5, pp. $1259-1272,2013$.

[25] J. Canny, "A computational approach to edge detection," Pattern Analysis and Machine Intelligence, IEEE Transactions on, vol. PAMI8, no. 6, pp. 679-698, Nov 1986.

[26] S. Suzuki et al., "Topological structural analysis of digitized binary images by border following," Comput. Vision, Graph., and Image Process., vol. 30, no. 1, pp. 32-46, 1985.

[27] S. Karaman and E. Frazzoli, "Sampling-based algorithms for optimal motion planning," Int. J. Robot. Res., vol. 30, no. 7, pp. 846-894, 2011

[28] M. Likhachev, "Search-based planning for large dynamic environments," Ph.D. dissertation, University of Southern California, 2005.

[29] D. Soetanto, L. Lapierre, and A. Pascoal, "Adaptive, non-singular pathfollowing control of dynamic wheeled robots," in Proc. 42nd IEEE Conf. Decision and Control, vol. 2. IEEE, 2003, pp. 1765-1770. 